\title{
Optimizing ultrasensitive single electron magnetometer based on nitrogen-vacancy center in diamond
}

\author{
WANG PengFei, JU ChenYong, SHI FaZhan \& DU JiangFeng* \\ Hefei National Laboratory for Physics Sciences at Microscale, and Department of Modern Physics, University of Science and Technology of \\ China, Hefei 230026, China
}

Received April 3, 2013; accepted May 28, 2013

\begin{abstract}
The measurement of the weak magnetic field in nanoscale resolution and at room temperature is always a significant topic in biological, physical, and material science. Such detection can be used to decide the characterization of the samples, such as cells, materials, and so on. Nitrogen-vacancy $(\mathrm{NV})$ center in diamond has been proved to be able to detect a magnetic field with nano Tesla sensitivity and nanometer resolution at room temperature. Here we experimentally demonstrate an optimized NV center based single electron magnetometer in a commercial diamond and under a home-built optically detected magnetic resonance (ODMR) microscope. With current technology, we change the optically detected time window to get a better signal to noise ratio, and use dynamical decoupling to increase the slope of magnetic field amplitude versus fluorescence signal. By employing the 8-pulse XY-4 dynamical decoupling sequence we achieve a sensitivity of $18.9 \mathrm{nT} / \sqrt{\mathrm{Hz}}$, which is 1.7 times better than spin echo. We also propose a NV center based scanning diamond microscope for electron and nuclear spins detection as well as nanoscale magnetic resonance imaging. If it is realized, the NV center based magnetometry will have wide application in the future.
\end{abstract}

nitrogen-vacancy center, diamond, magnetometer

Citation: Wang P F, Ju C Y, Shi F Z, et al. Optimizing ultrasensitive single electron magnetometer based on nitrogen-vacancy center in diamond. Chin Sci Bull, 2013, 58: 2920-2923, doi: 10.1007/s11434-013-5967-z

In the past decade [1], due to well understanding of the properties, nitrogen-vacancy (NV) center has been a good candidate for building the scalable quantum computer, with several quantum computation gates and algorithms demonstrated on it $[2,3]$. Since it has properties of long coherence time, good optical property, and solid-state attribute, NV center is also a potential magnetometer sensor with high sensitivity, high resolution and can work at room temperature $[4,5]$. Related work has demonstrated it has the ability of detecting weak magnetic field with sensitivity as low as $4 \mathrm{nT} / \sqrt{\mathrm{Hz}}$ [6]. In addition, since $\mathrm{NV}$ center can be placed less than $10 \mathrm{~nm}$ close to the sample, it can do measurements with nanoscale resolution [7-9]. All these advantages mentioned above make the NV center based magnetometer very powerful. Recent work has shown many of its applications in sensing, such as sensing remote nuclear spin clusters [10]

*Corresponding author (email: djf@ustc.edu.cn) and internal single nuclear spins [11-13], detecting external electron spin ensemble [14], and scanning diamond magnetic sensor $[8,15]$. With the minimum nano Tesla sensitivity, NV center has been developed to be a good candidate of a universal sensor for single electron spin, single molecule, as well as single nuclear spin detection and imaging.

NV center consists of a substitutional nitrogen atom and a neighboring vacancy which traps two unpaired electrons. The strong electron-electron coupling leads to a singlettriplet structure. Its triplet ground state has a zero splitting of $D=2.88 \mathrm{GHz}$. The principle of a NV center based quantum magnetometer is as follows: Firstly, the spin is prepared on one of the superposition state $|0\rangle+| \pm 1\rangle$. Then during the evolution with the weak magnetic field, the superposition state will accumulate a relative phase on $|0\rangle$ and $| \pm 1\rangle$. Finally we map the phase to probability on $|0\rangle$ and read out the result [4]. The sensitivity is related to the 
detected signal and evolution time. For a given sine wave AC magnetic field with a frequency of $v=1 / \tau$, it can be written as

$$
\eta \propto \frac{\sigma}{P_{\mathrm{B}}^{\prime}} \cdot \sqrt{\tau}
$$

where $P_{\mathrm{B}}^{\prime}$ is the maximum slop of the magnetometer fluorescence signal $P_{\mathrm{B}}=\left[1+F(\tau) \cos \left(2 \tau g \mu_{\mathrm{B}} B_{\mathrm{AC}} / \pi \hbar\right)\right] / 2$ varying with the weak magnetic field amplitude $B_{\mathrm{AC}} . F(\tau)$ is the fluorescence signal without weak magnetic field, $g$ is the $g$-factor of NV center, and $\mu_{\mathrm{B}}$ is the Bohr magneton [4]. To optimize the performance of NV center based magnetometer, several works have been done to improve its sensitivity. Since the sensitivity is proportional to the square root of coherence time, a ${ }^{12} \mathrm{C}$ enriched ultrapure diamond was made to prolong $T_{2}$ to $1.8 \mathrm{~ms}$ and thus a sensitivity of $4 \mathrm{nT} / \sqrt{\mathrm{Hz}}$ was achieved in [7]. In [16], multi-sequence dynamical decoupling was proved to be able to apply for improving the sensitivity effectively. In addition, the other thing is to minimize the standard deviation $\sigma$, as well as increase the signal to noise ratio (SNR). Obviously, $\sigma$ is proportional to the sensitivity according to eq. (1). And SNR is limited by the photon collecting efficiency, which is typically $0.1 \%$ due to the normal ODMR setup and commercial sample. In this work we will demonstrate a systematic method to increase the SNR by optimizing the laser readout time sequence. Combining with the dynamical decoupling [17-20], we will excavate the potentiality of building a NV based magnetometer by using our setup and discuss the application of the optimized magnetometer in scanning diamond probe microscope.

The axis of NV center is parallel to [111] crystallographic axis of the diamond. We used a commercial ultrapure bulk diamond from Element Six with a nitrogen concentration below $5 \mathrm{ppb}$. The diamond was placed under a permanent magnet to generate a Zeeman splitting of $\sim 115 \mathrm{MHz}$. A single copper coil was placed to generate a weak magnetic field on the [111] axis of diamond (Figure 1(a)). The whole setup was placed in a room with the temperature having a stability of $\pm 0.5 \mathrm{~K} / \mathrm{h}$ in order to minimize the magnetic field drift. To locate the NV center, firstly we scanned the sample by nanometer resolution scanner to find some photon emitters. Then, the autocorrelation function $g^{2}(\tau)$ was measured to ensure the photons are emitted from a single quantum emitter (Figure 1(b)). Finally, the continuous wave spectrum was acquired to confirm that this emitter was a single NV center and its axis direction was along the static magnetic field.

We employed a low noise $532 \mathrm{~nm}$ laser pulse with a length of $2 \mu$ s to initialize the NV to $m_{s}=0$ with a probability higher than $95 \%$ [1]. This laser was also used to read out the spin state. We collected the fluorescence photons whose (a)

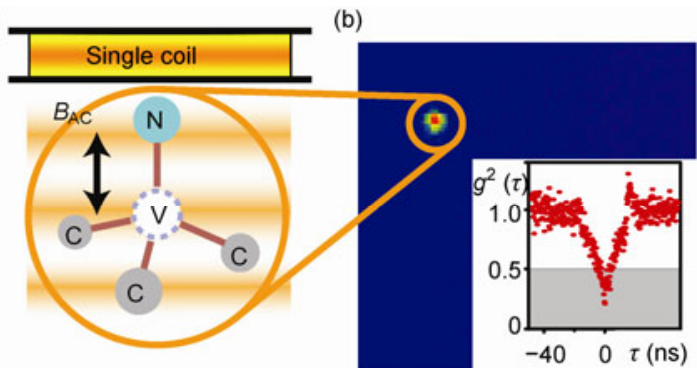

(c)

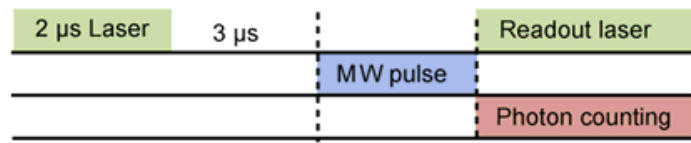

Optical and microwave pulse sequence

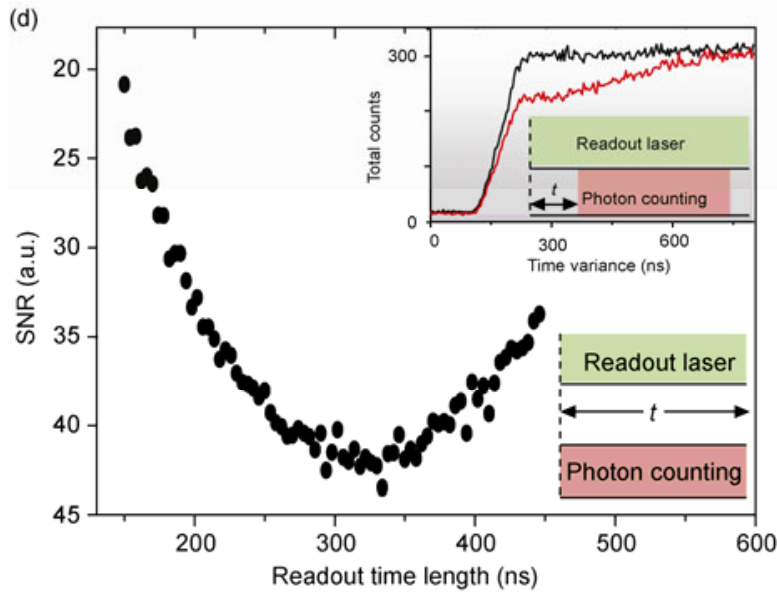

Figure 1 (a) NV center is placed in a permanent magnetic field and a weak AC magnetic field $B_{\mathrm{AC}}$; (b) scanning fluorescence image and autocorrelation function. The density of the NV center is very low because of the low nitrogen concentration. The dip value is below 0.5 , which indicates this is a single photon emitter; (c) pulse sequence for NV center experiments. $2 \mu$ s laser followed by $3 \mu$ s wait time initializes the NV center to $m_{s}=0$ state. In the end, the laser and photon counting channel are opened simultaneously to read out the probability on $m_{s}=0$; (d) to optimize the readout time sequence, we did series of measurements. We measured the SNR versus the time length of readout. The top-right inset is how we derived the initialization curve of the observed NV center. We fixed the photon counting time length and changed its synchronization time with laser. By comparing the total counts of $m_{s}=0$ with $m_{s}=1$, we know the polarization time is about $600 \mathrm{~ns}$.

wavelength is between 650 and $775 \mathrm{~nm}$. When the NV center was prepared to $m_{s}=1$ state, there was a procedure that the probability on $m_{s}=0$ was changed from 0 to 1 slowly under laser illumination (Figure 1(d) inset). When we changed the readout time sequence, the contrast of photon counts between $m_{s}=0$ and 1 varied. This variation affected the SNR (Figure 1(d)). Increasing readout time length would make a smaller deviation but the contrast is also reduced, while decreasing the readout time length would make a larger contrast but the photon number was less and the deviation became bigger. By using the SNR versus readout time length curve we could get an optimal tradeoff. In the experiment, we optimized the SNR in this way and 
got an optimal readout time length of 330(10) ns.

To improve the sensitivity, we also adopted a multi-pulse dynamical decoupling. In NV center based magnetometer, periodic multi-pulse dynamical decoupling can not only prolong the coherence time, but also make the relative phase accumulated $(N+1) / 2$ times than Hahn echo. Then the fluorescence signal will be

$$
P_{\mathrm{B}}=\left[1+F_{\mathrm{dd}}(N \tau) \cos \left(2 N \tau g \mu_{\mathrm{B}} B_{\mathrm{AC}} / \pi \hbar\right)\right] / 2,
$$

where $F_{\text {dd }}(N \tau)$ is the bare fluorescence signal under dynamical decoupling. The sensitivity will be

$$
\eta \propto \frac{\sigma}{P_{\mathrm{B}}^{\prime}} \cdot \sqrt{2 N \tau} .
$$

Considering the signal decay caused by decoherence, we can find the value of sensitivity is decreased by $F_{\text {dd }}(N \tau) \sqrt{N} / F(\tau)$ times.

In the experiment, firstly, we measured the coherence decay curve. In 41 Gauss static magnetic field, there existed coherence collapses and revivals due to ${ }^{13} \mathrm{C}$ spin bath at about $41 \mathrm{kHz}$ [1]. In the collapses, there was no coherence in NV center so $F(\tau)=0$. The sensing should avoid these collapses. As a demonstration, we set the weak AC magnetic field to $v=3.7313 \mathrm{kHz}$ to match one of the maximum revival times, which is $268 \mu \mathrm{s}$. The AC magnetic field was synchronized with the spin echo and the phase was set to 0 for best performance. In the dynamical decoupling sequence, we used XY-4 pulses, which was the best multi-pulse dynamical decoupling sequence for NV center so far $[19,20]$. A 34 ns $\pi$ pulse was put into use to acquire a rotating fidelity higher than $96 \%$. The 8 pulses XY-4 sensing signal amplitude was smaller than spin echo but the slop was larger. Figure 2 shows our main result of this paper. We got a sensitivity of $\eta_{\mathrm{dd}}=18.9 \mathrm{nT} / \sqrt{\mathrm{Hz}}$ while $\eta_{\mathrm{se}}=31.3 \mathrm{nT} / \sqrt{\mathrm{Hz}}$ for the spin echo. An improvement of 1.7 times was achieved by appling the dynamical decoupling. The maximum decoupling pulse number was 8 and increasing the number of decoupling pulses did not further prolong decoherence time. We think it is limited by the polarization time $T_{1}$ (Figure 3).

With an averaged time of $1000 \mathrm{~s}$, a magnetic field $0.6 \mathrm{nT}$ was measured by our optimized magnetometer. This value corresponds to the magnetic field generated by a single electron spin at $40 \mathrm{~nm}$ away or a single nuclear spin at 4.4 $\mathrm{nm}$ away. This result enables NV center to be a good candidate for the probe to detect single electron and nuclear spin $[21,22]$. In Figure 4 we show a new scanning diamond microscopy proposal for single electron spin detection and nanoscale magnetic resonance imaging (nano MRI). The probe is made of diamond with $\mathrm{NV}$ center close to the surface. The depth of the NV center is below $10 \mathrm{~nm}$ [23]. This distance can avoid the strong depolarization effect due to the diamond surface and reach a decoherence time of more (a)

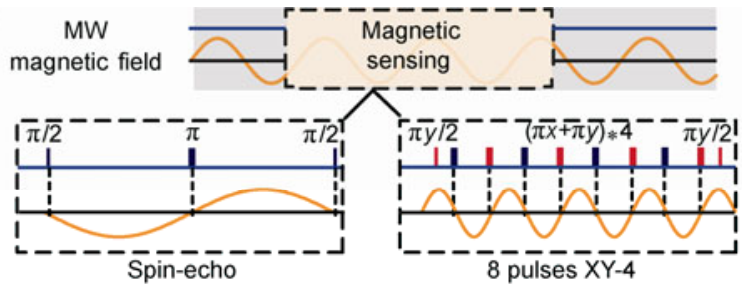

(b)

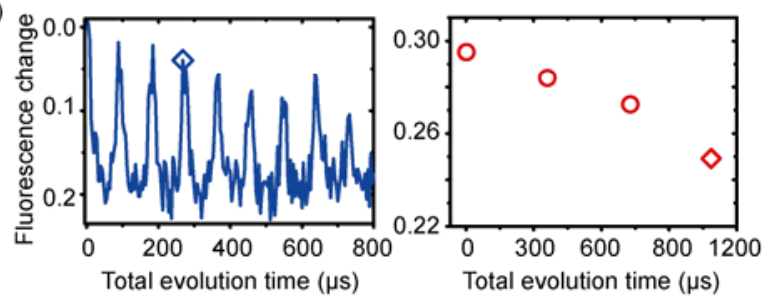

(c)

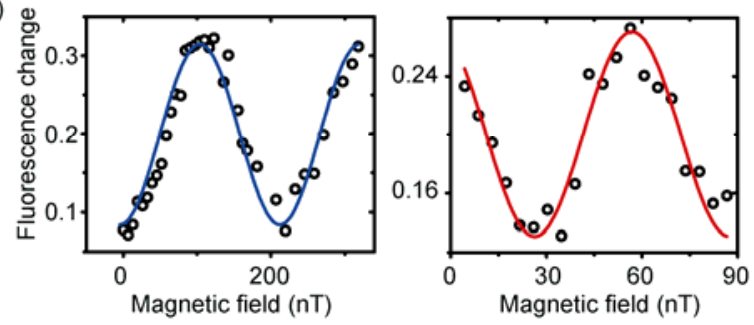

Figure 2 The pulse sequence and experiment result. (a) Pulse sequence for magnetic sensing. The yellow curve is weak magnetic field waveform; (b) spin echo and 8-pulse XY-4 signal. The blue curve is spin echo, while the red circles and diamond are XY-4. In XY-4, we only measured the highest points of the revival for saving time. The blue and the red diamond are the time points chosen to do the magnetic field sensing; (c) fluorescence change of the NV center based magnetometer versus weak AC magnetic field $B_{\mathrm{AC}}$ in spin echo (left) and 8-pulse XY-4 sequence (right). All of the data is averaged by $10^{5}$ times for increasing SNR.

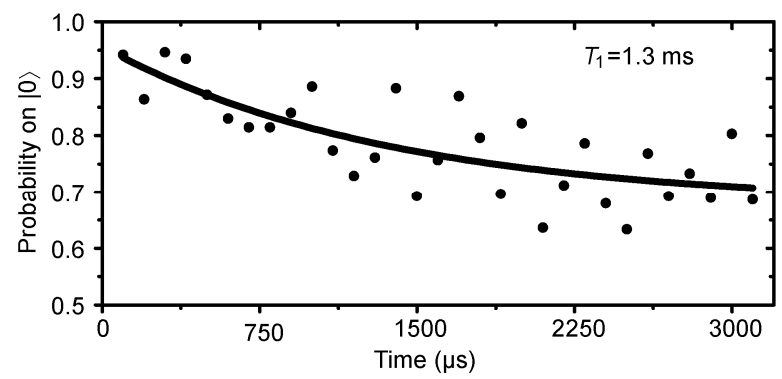

Figure 3 Correlation between probability and evolution time. To clearly show the depolarization time, we normalized the data by signal $|0\rangle$ and $|1\rangle$. By changing time interval between two laser pulses, we obtained this curve. The point is fitted with an exponential decay and the decay time is about $1.5 \mathrm{~ms}$.

than $70 \mu$ s [15]. We adopt multipulse double electronelectron resonance (DEER) or electron-nuclear double resonance (ENDOR) for the detection [24]. In this protocol, we use microwave $\pi$ pulses to manipulate the sample spins periodically and synchronize them with the NV center's $\pi$ pulses. The sample spins act similarly like a weak AC magnetic field source. Then there is a relative phase on NV center superposition state. By changing the time interval between each $\pi$ pulse, we can get a curve of fluorescence 


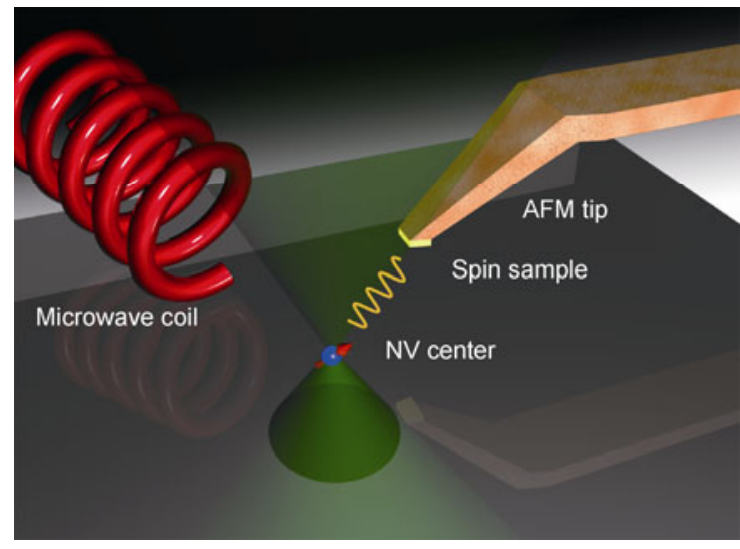

Figure 4 Proposal image for scanning diamond microscopy. A sample is mounted on the tip of an atomic force microscope (AFM). A coil is used for microwave radiation. The sample and the NV center are coupling by dipole-dipole interaction.

signal versus relative phase $[21,24]$. Comparing this curve with that of the bare NV center, we will know whether there are electron spins or nuclear spins nearby. Besides, we can also do nano MRI by scanning the sample around the NV center.

In conclusion, we discussed the important influencing factors to the sensitivity of the NV based magnetometer. We experimentally optimized the NV based magnetometer and improved its sensitivity. Finally, we proposed a scanning diamond microscope for electron spin detection and nanoscale magnetic resonance imaging based on the current technology. Once such microscopy is realized, we believe the NV center based magnetometry will have a wide application.

This work was supported by the National Basic Research Program of China (2013CB921800), the National Natural Science Foundation of
China $(11227901,91021005,10834005,11104262)$ and the Chinese Academy of Sciences.

1 Childress L, Dutt M V G, Taylor J M, et al. Science, 2006, 314 : 281-285

2 Shi F Z, Rong X, Xu N Y, et al. Phys Rev Lett, 2010, 105: 040504

3 Xu X K, Wang Z X, Duan C K, et al. Phys Rev Lett, 2012, 109: 070502

4 Maze J R, Stanwix P L, Hodges J S, et al. Nature, 2008, 455: 644-647

5 Taylor J M, Cappellaro P, Childress L, et al. Nat Phys, 2008, 4: $810-816$

6 Balasubramanian G, Neumann P, Twitchen D, et al. Nat Mater, 2009, 8: 383-387

7 Balasubramanian G, Chan I Y, Kolesov R, et al. Nature, 2008, 455: 648-651

8 Grinolds M S, Maletinsky P, Hong S, et al. Nat Phys, 2011, 7: 687-692

9 Ofori-Okai B K, Pezzagna S, Chang K, et al. Phys Rev B, 2012, 86: 081406

10 Zhao N, Hu J L, Ho S W, et al. Nat Nanotech, 2011, 6: 242-246

11 Zhao N, Honert J, Schmid B, et al. Nat Nanotech, 2012, 7: 657-662

12 Kolkowitz S, Unterreithmeier Q P, Bennett S D, et al. Phys Rev Lett, 2012, 109: 137601

13 Taminiau T H, Wagenaar J J T, Sar T V D, et al. Phys Rev Lett, 2012, 109: 137602

14 Mamin H J, Sherwood M H, Rugar D. Phys Rev B, 2012, 86: 195422

15 Maletinsky P, Grinolds M S, Hong S, et al. Nat Nanotech, 2012, 7: 320-324

16 De L G, Riste D, Dobrovitski V V, et al. Phys Rev Lett, 2011, 106: 080802

17 Du J F, Rong X, Zhao N, et al. Nature, 2009, 461: 1265-1268

18 Huang P, Kong X, Zhao N, et al. Nat Commun, 2011, 2: 570

19 De L G, Wang Z H, Ristè D. Science, 2010, 330: 60-63

20 Ryan C A, Hodges J S, Cory D G. Phys Rev Lett, 2010, 105: 200402

21 Mamin H J, Kim M, Sherwood M H, et al. Science, 2013, 339: $557-560$

22 Staudacher T, Shi F Z, Pezzagna S, et al. Science, 2013, 339: 561-563

23 Ofori-Okai B K, Pezzagna S, Chang K. Phys Rev Lett, 2012, 86: 081406

24 Grinolds M S, Hong S, Maletinsky P, et al. Nat Phys, 2013, 9: 215-219

Open Access This article is distributed under the terms of the Creative Commons Attribution License which permits any use, distribution, and reproduction in any medium, provided the original author(s) and source are credited. 